Quaderni di Geografia Cahiers de Gécongaty rataphie

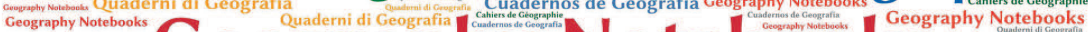
Cuadernos de Geografia Geography Notebooks $U 201000$ Cahiers de Géographi Cahiers de Géographie cahiers de Géographie Cuadernos de Geografía Cahiers de Géographie Cahiers de Géographie Cuadernos de Geografía Geography Notebooks

\author{
$4(2021)$ \\ 1 \\ Teatro di suoni. \\ Spazi acustici teatrali e territoriali
}

A cura di

Martino Mocchi, Lorena Rocca, Demis Quadri and Carlotta Sillano

EDITORIAL

Teatro di suoni per l'attaccamento ai luoghi. Uno sguardo geografico 11

Lorena Rocca

Per un teatro di suoni. Riflessioni su possibili dimensioni sonore nelle 23 creazioni site-specific di physical theatre

Demis Quadri

INTRODUCTION

Teatro di suoni. Spazi acustici teatrali e territoriali

Demis Quadri e Lorena Rocca

SPECIAL Issue

I suoni di Mantova come strumenti di interpretazione del paesaggio.

Tra turismo sostenibile ed educazione al patrimonio culturale

Valeria Pecorelli, Franca Zuccoli, Alessandra De Nicola, Enrico Squarcina

Il paesaggio sonoro campano tra contemporaneità e nuove forme

di progettualità turistica

Germana Citarella 
La narrazione spettacolarizzata del paesaggio sonoro.

Da Giuseppe Chiari a Philip K. Dick e oltre

Francesco Michi

Musica di paesaggi sonori. Enunciazione, risignificazione, comunicazione

Carlotta Sillano

Camminare per ascoltare. Partiture invisibili del territorio abitato

Elisabetta Senesi

Il paesaggio sonoro in relazione. Suono, movimento e immagini per stimolare complessità percettiva Angela Calia

Groove Fields. Understanding the Dance Floor from an Art-Based Research Perspective

Sebastian Mattbias

Il silenzio come esperienza trasformativa. L'importanza del silenzio nella meditazione e in ambito professionale

Sebastiano Caroni

Progettare il silenzio. Una lettura acustica dell'ex villaggio sanatoriale 125 Morelli a Sondalo

Martino Mocchi

Voicing One's Will. Theatre as Audio-Visual Hypotyposis of the Poetic

Michael Groneberg

Music and Clowning in Europe, 20th-21st centuries

Anna Stoll Knecht

Il paesaggio sonoro nella composizione musicale. Un percorso bibliografico

Stefano Alessandretti

\#exploreART: il labirinto di A. Pomodoro e i bambini. Un progetto di fruizione condivisa con percorsi sensoriali partecipati Alessandra De Nicola, Franca Zuccoli 


\section{OTHER EXPLORATIONS}

Il rumore lontano. Intervista a Lorena Rocca

a cura di Martino Mocchi

Re Cervo. Intervista a Antonella Astolfi

a cura di Krizia Bonaudo e Demis Quadri

Centovalli-Centoricordi. Intervista a Oliviero Giovannoni a cura di Krizia Bonaudo e Demis Quadri

Alla ricerca di un metodo: Open Space Technology 



\title{
Progettare il silenzio. Una lettura acustica dell'ex villaggio sanatoriale Morelli a Sondalo
}

\author{
Martino Mocchi \\ Dipartimento ABC, Politecnico di Milano; Dipartimento DFA, SUPSI \\ DOI: https://doi.org/10.7358/gn-2021-001-moc1
}

\begin{abstract}
The sanatorium Morelli was built in Sondalo during the fascist era (1932-1940). The strong visual impact of the structure is counterbalanced by a delicate acoustic approach, made necessary by the need to create silent places for supporting medical treatments. The project of "silence" is not only the result of a punitive stance, which simply intends to reduce sound emissions, but above all the effect of a positive attitude, aiming at selecting specific sounds, in accordance with the social and environmental characteristics of place. This produces the sense of participation and identification of the users, generating collective cares and protection. That of Morelli, indeed, is a relevant lesson for the contemporary reflection, with a view to finding good strategies for soundscape design.
\end{abstract}

Keywords: soundscape design; silence; Morelli sanitarium; fascist architecture; care.

Parole chiave: progetto del paesaggio sonoro; silenzio; villaggio sanatoriale Morelli; architettura fascista; cura.

\section{Avvio}

Calma notte serena, tu non sai

il fascino che l'ore t'han portato.

Io ti sorprendo

da una fredda ed estranea terrazza 
e si a lungo ti guardo

che pari infastidita.

Oltre non odo

che respiri malati

ed il lontano correre d'un auto.

Ora nei corridoi,

fatti più lunghi dal silenzio e i viali

uno cammina e vigila - il dolore -

che pare tutti noi.

I versi del poeta valtellinese Giulio Pedranzini ${ }^{1}$, scritti negli anni trascorsi all'interno del Morelli, dimostrano chiaramente l'importanza dell'elemento sonoro nell'esperienza della vita sanatoriale. I "respiri malati" e il "lontano correre d'un auto" si staccano come momenti isolati, quasi drammatici, all'interno di quella che viene descritta come 1'“impronta sonora" (Schafer 1985) più caratterizzante del paesaggio: il silenzio. Un elemento tradizionalmente considerato assenza, negazione, mancanza di suono, che assume invece in questo contesto il ruolo di una presenza viva, talmente forte da influenzare la stessa percezione dello spazio e del tempo. Diluendo, rarefacendo, allungando una sequenza ripetitiva che diventa espressione più evidente del "dolore" che sta alla base della condizione del degente.

\section{IL SANATORIO, UNA “MACCHINA” PER PRODURRE SILENZIO}

L'origine del concetto di sanatorio prende forma attorno alla metà dell'Ottocento, per far fronte all'ampia diffusione di malattie croniche a lunga degenza, fra tutte quella che è stata definita la "piaga del secolo", la tubercolosi. Vista l'assenza di antibiotici e soluzioni farmacologiche efficaci per queste patologie, il percorso di cura si sviluppa secondo un modello complesso, che punta al riequilibrio generale tra la condizione dell'individuo e quella dell'ambiente. Spostandosi dalla metropoli indu-

${ }^{1}$ Sulla base delle mie informazioni, la poesia non è pubblicata all'interno di antologie o volumi. È stata presentata durante una serata di riflessione intitolata "La magia del Morelli nelle poesie di Pedranzini”, tenutasi il 20 luglio 2012 presso il Presidio Morelli di Sondalo. La serata è stata organizzata dall'Azienda Ospedaliera Valtellina e Valchiavenna, in collaborazione con il Comune di Sondalo e le Associazioni Terra Celeste e Pro Valtellina. 
strializzata, caratterizzata da inquinamento, traffico, ritmi di vita e di lavoro insostenibili, il degente si trova immerso in un contesto naturale con aria pura, abbondanza di luce, una tipologia di vita più distesa per conciliare il riposo e la riflessione. Respirare, mangiare, dormire sono elementi fondamentali per la guarigione, che permettono di ricostruire un equilibrio fisico e psichico (Bonesio et al 2012).

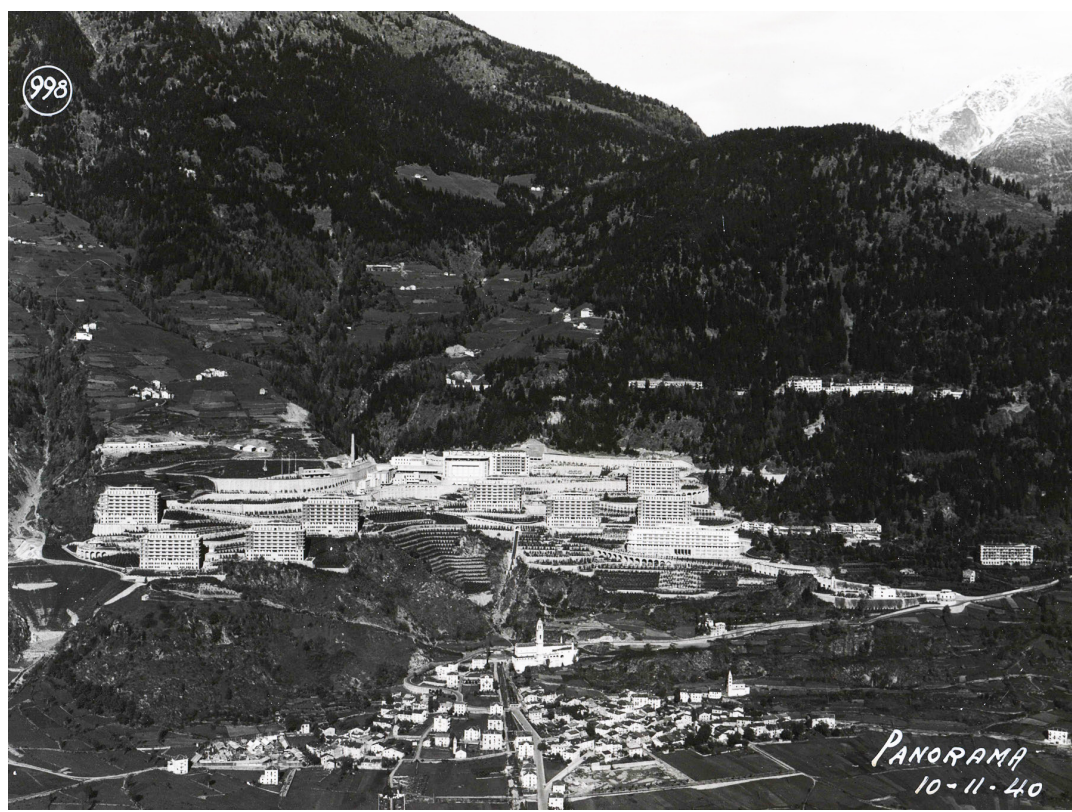

Figura 1. - Vista del versante sud di Sondalo con il cantiere del villaggio Morelli, 1940. Fonte: Archivio Morelli.

Per realizzare tali condizioni, il sanatorio si strutturava come una gigantesca "macchina" ${ }^{2}$, collocata in zone di pregio ambientale, in grado di ospitare anche centinaia di pazienti in modo residenziale, dando vita a veri e propri piccoli villaggi o micro-città, all'interno dei quali gli individui costruivano amicizie e stabilivano relazioni anche stabili. I lunghi

2 Questa definizione richiama alla mente il concetto foucaultiano di "dispositivo" (Foucault 1993), accomunando quindi il sanatorio ad altri luoghi come prigioni e scuole. In particolare, in accordo con l'interpretazione data da Deleuze e poi da Agamben a questo concetto, risulta interessante segnalare la relazione tra dimensione architettonica, pratiche sociali e ideologia della cura favorita all'interno di questi contesti. 
periodi di degenza, che duravano in media diversi mesi, conferivano al sanatorio un'immagine distante da quella del moderno ospedale - in quanto struttura meramente assistenziale - caratterizzandolo per una dimensione relazionale, conseguente a una necessaria presa di distanza dalle abitudini sociali e ambientali sedimentate.

Tra le strutture sanatoriali del secolo scorso, una delle più significative è certamente quella del Morelli (Fig. 1), realizzata a Sondalo in piena epoca fascista, tra il 1932 e il 1940, con l'obiettivo dichiarato di realizzare il più grande sanatorio europeo (Bonesio et al 2011). La dimensione della struttura è imponente, arrivando a ospitare fino a 3.000 degenti, a cui si aggiungevano altrettanti utenti - medici, infermieri, personale di servizio. Un vero e proprio borgo, quindi, costruito con linguaggio potente e monumentale, per dare impulso a quella che il Regime e l'Istituto Nazionale Fascista di Previdenza Sociale avevano definito una necessaria "bonifica del popolo".

Dal punto di vista estetico-paesaggistico, il Morelli interviene in modo deciso all'interno del contesto, piegando alle proprie necessità un intero versante della montagna e ridefinendo il significato paesaggistico di tutta l'alta Valtellina. L'assenza di compromesso nel disegno e nella concezione emerge anche dalla scelta delle soluzioni tecnico-costruttive: dalla simbolica galleria che introduce all'interno del complesso agli enormi terrazzamenti, su cui prendono forma i padiglioni con un carattere quasi metafisico.

A uno sguardo più attento, il Morelli rivela dei tratti più sofisticati. Passeggiare all'interno del parco permette di cogliere il legame tra intento celebrativo e attenzione per il dettaglio, a vantaggio di ambienti diversificati che possono soddisfare le diverse esigenze dell'utenza. La selezione della vegetazione è curata, pensata per massimizzare la qualità ambientale dei luoghi, rispettando al contempo il contesto naturalistico esistente. Le perimetrazioni sono realizzate con pietra locale, senza introdurre elementi invasivi. L'utilizzo delle differenze di quota e di dispositivi light-come i pergolati - orienta il pubblico verso una specifica fruizione delle aree, pur nel rispetto dei caratteri locali. Lo stesso approccio si trova nella costruzione dei padiglioni: basti pensare alla scelta cromatica degli edifici, in continuità con le tinte e le tonalità atmosferiche di questo tratto alpino.

La "titanica sfida" che il Morelli lancia alla montagna (Bonesio 2012), e l'apparente sfrontatezza del suo disegno, si rivela quindi come il prodotto di un più articolato pensiero tecnico-progettuale, sostenuto da 
una chiara visione paesaggistica, compositiva e culturale dell'ambiente e della società ${ }^{3}$.

\section{Il PROGETTO DEL SILENZIO}

Le caratteristiche acustiche del Morelli risultano quantomai istruttive: è proprio il contrasto tra visivo e sonoro, infatti, a mostrare nella maniera più radicale la complementarità tra le necessità monumentali e celebrative dell'impianto e la ricerca di un'assenza che, con un solo termine, si potrebbe definire "silenzio". La forza visiva con cui il villaggio si impone alla montagna fa da contraltare alla delicatezza e al gentile accoglimento che l'impianto stabilisce con il paesaggio sonoro esistente. In termini acustici, il Morelli si appoggia al contesto senza alterarne le dinamiche, cercando di "non fare rumore". La progettazione degli spazi comuni, la cura dei percorsi viabilistici, la disposizione periferica degli edifici con i servizi rappresentano alcuni degli accorgimenti chiaramente pensati per ridurre l'impatto sonoro della struttura ${ }^{4}$.

Tra i molteplici elementi che orientano tale lettura bisogna indivi-

${ }^{3}$ Una lezione che risulta estremamente attuale ancora ai giorni nostri, ponendosi in antitesi rispetto alla tendenza al "nascondimento" che ha guidato, per esempio, l'insediamento nella bassa Valtellina. Un permissivismo sorretto da una (mancanza di) visione politica, che ha fatto sorgere decine e migliaia di piccoli capannoni, stabilimenti, depositi affiancati tra loro - rigorosamente protetti alla vista da elementi "verdi" - che hanno di fatto deturpato larga parte del paesaggio, sia in termini estetici che culturali. La concentrazione a cui aspira il Morelli è invece finalizzata alla realizzazione di una immagine ben chiara e definita, che si assume le proprie responsabilità senza compromessi, dando vita a una precisa concezione della società e dell'ambiente. All'interno del villaggio nulla è casuale, ogni dettaglio è pensato e valutato scrupolosamente, realizzato per rispondere a specifiche esigenze mediche e collettive.

${ }^{4}$ Le caratteristiche del Morelli qui descritte traggono origine da una lettura esperienziale-fenomenologica personale, maturata in una serie di visite tra il 2012 e il 2016, all'interno di una ricerca coordinata dalla prof. Luisa Bonesio con oggetto "Il paesaggio e le architetture del Villaggio sanatoriale Morelli di Sondalo”. In questo contesto, l'autore ha partecipato come guida al ciclo di visite "Il paesaggio e le architetture del Villaggio Morelli" (2012 e 2013), all'organizzazione delle mostre "Dal mal sottile alla tubercolosi resistente" (2013), "Il villaggio incantato. Vie dello sguardo" (Ugo Locatelli 2014). È stato protagonista di alcuni eventi all'interno del Museo dei Sanatori, come relatore e attraverso la produzione di materiali audio dedicati. La scelta metodologica, in accordo con altre esperienze condotte in differenti contesti (Mocchi 2020), si basa sulla convinzione che sia proprio l'affinamento della sensibilità percettiva individuale a rendere possibile la comprensione dei tratti profondi e multisensoriali alla base del senso dei luoghi. 
duare l'imponente operazione di terrazzamento alla base della costruzione del complesso (Fig. 2). La rottura della costa alpina svolge un forte effetto di diffrazione delle onde sonore, limitando la penetrazione del suono della valle all'interno delle aree di degenza. L'effetto è chiaramente percepibile stando sul bordo dei terrazzamenti, dove si nota un significativo abbassamento del volume, anche solo spostandosi di pochi metri verso l'interno. Le esigenze primariamente costruttive e funzionali che motivano tale intervento sono quindi interpretabili anche in senso acustico, specialmente in funzione della tranquillità degli spazi comuni al piano terreno degli edifici.

Risulta poi interessante porre attenzione su quegli espedienti pensati per svolgere un ruolo di mitigazione acustica rispetto al rumore delle attività interne. Le teleferiche che collegano il padiglione dei servizi con quelli destinati alla degenza sono un esempio significativo, che rende evidente il tentativo di limitare il movimento, pur mantenendo vivi i collegamenti per la distribuzione puntuale dei pasti, delle lenzuola, lo scambio di altri strumenti dal padiglione centrale a quelli che ospitano i pazienti.

A questi elementi “negativi” è bene accostare altre strategie impiegate per costruire attivamente l'esperienza acustica collettiva.

Il primo elemento è proprio l'ingresso al sanatorio: la monumentale galleria che non ha alcuna utilità strettamente viabilistica o funzionale, ma è pensata per segnare l'ingresso dall'esterno della montagna. I due lati sono fortemente differenziati proprio da un passaggio percettivo, dove il mutamento visivo è completato da una altrettanto radicale trasformazione del contesto acustico. La crescita del riverbero arriva a produrre una distorsione del suono, generando un effetto di caos che si risolve non appena entrati nel parco. Ciò assume un significato simbolico, a rappresentare il contrasto tra il mondo esterno e il mondo interno: un abbandono del "rumore" che introduce al "silenzio".

Vi è poi la campana della chiesa, unico dispositivo acustico in grado di comunicare in tutta l'area, che risulta misurata ed equilibrata, producendo un suono percepibile ma al contempo non invasivo rispetto all'ambiente circostante. Il che manifesta, allo stesso tempo, la volontà di restituire al suono un ruolo di regolazione temporale dell'attività umana.

Va quindi sottolineato l'impianto di filodiffusione presente nell'intero villaggio, a cui ogni paziente poteva connettersi attraverso cuffie audio fornite in dotazione. La presenza di una radio locale, con musica e informazioni appositamente studiate per la vita sanatoriale, riflette l'idea futurista secondo cui il suono può essere un elemento attivo per guidare e orientare la disposizione emozionale dell'uomo nelle varie fasi del 

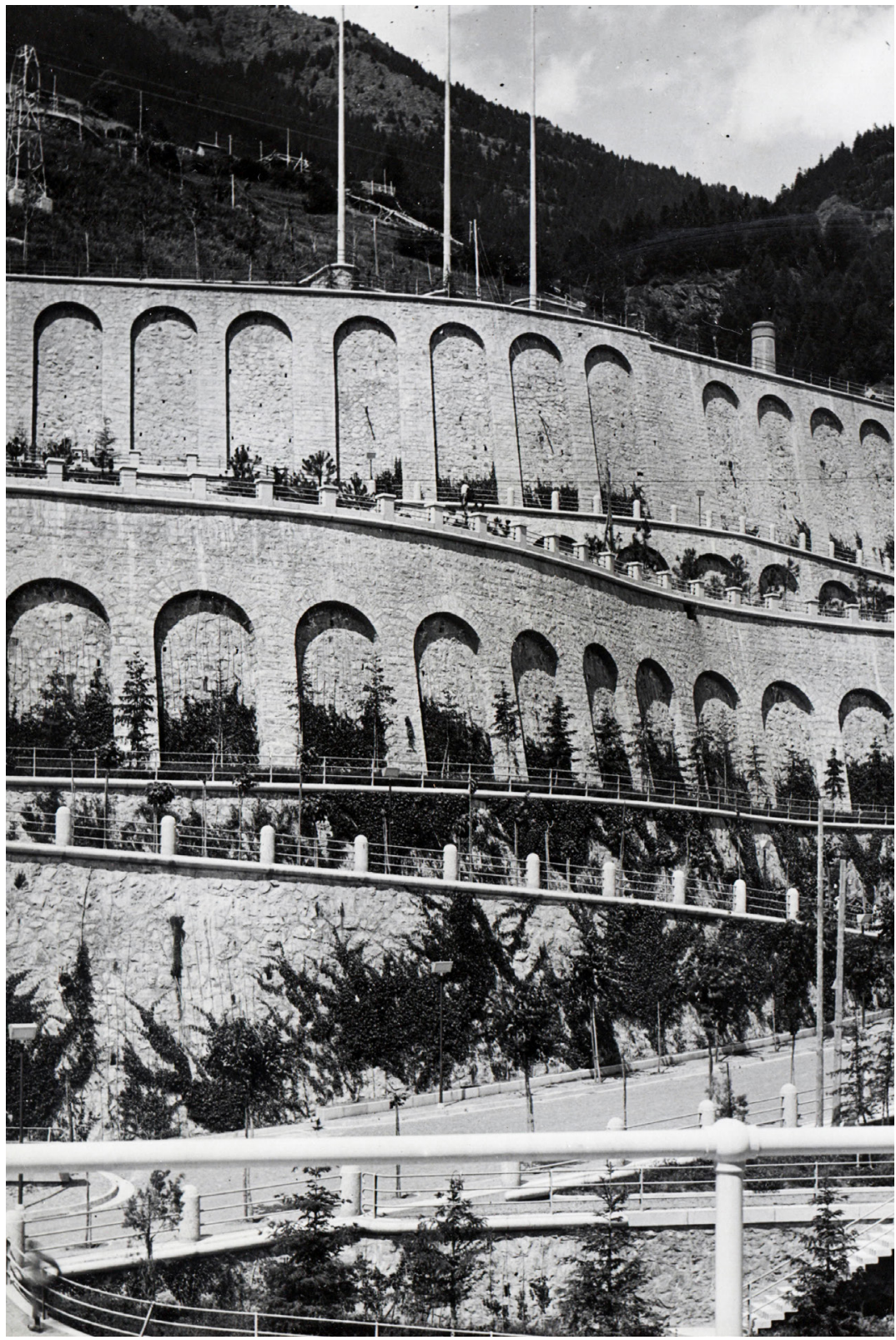

Figura 2. - Muri di contenimento dei terrazzamenti, 1940. Fonte: Archivio Morelli. 
giorno ${ }^{5}$.

Infine, è lo stesso parco a svolgere un ruolo di selezione e costruzione dell'esperienza acustica del villaggio. Attraverso la piantumazione di essenze vegetali e floreali, e grazie alla definizione di precisi intervalli tra gli elementi, il parco permette alla natura di entrare all'interno del complesso producendo un effetto ristoratore. Il suono degli insetti, degli uccelli, lo stormire del vento tra le fronde definiscono un paesaggio di quiete, in una armonia con l'intorno naturale. Emblematico di questo atteggiamento è il trattamento del ruscello che scorre nel mezzo del parco - il Rio - che in alcuni punti viene interrato, in altri lasciato libero, in altri ancora trattato con cascatelle artificiali per generare effetti acustici.

Tale breve lettura del parco del Morelli indica quindi una strada da seguire, basata sulla possibilità di coniugare il controllo e la mitigazione quantitativa del dato sonoro a una sua considerazione più puntuale e qualitativa. La silenziosità dell'esperienza sanatoriale è costruita a partire da una comprensione specifica della relazione tra suono umano e contesto fisico e culturale in cui si manifesta, alla luce di specifiche esigenze abitative e di cura. Il silenzio, dunque, come elemento non riducibile alla usuale definizione negativa di "assenza" (di suono, musica, rumore), ma risultato di una mediazione complessa, che coinvolge il livello percettivo, emotivo e simbolico del vivere e dell'abitare (Fig. 3).

\section{Riflessione CONClusiva}

Se da un lato è noto che "il silenzio non esiste" (Cage 1985), dall'altro è indubbio che questo termine si trovi disseminato nel linguaggio quotidiano (Filiberti 2011), rimandando a un orizzonte di senso apparentemente condiviso. Nella società contemporanea, il concetto si carica di un significato prevalentemente positivo, facendosi testimone dell'idea di evasione da un presente dominato da "rumore" (Dorfles 2008; Pivato 2011). Silenzio come elemento "accogliente", quindi, che supera la connotazione "oppressiva" che potrebbe legarsi ad altre disposizioni emotive quali malinconia, solitudine, tormento e disagio (Volcler 2012).

${ }^{5} \mathrm{Si}$ veda in particolare un tardo manifesto, scritto proprio durante gli anni della costruzione del Morelli (1933), intitolato "Manifesto futurista per la città musicale": «La musica ha influenze e risonanze non trascurabili sul nostro spirito», tanto che «il nostro lavoro, le nostre opere, le nostre creazioni sono tutte sospinte da una musica interiore» (Lombardi 2003, 118).

Geography Notebooks - 4 (2021) 1

https://www.ledonline.it/Geography-Notebooks/ 
È sempre più la contrapposizione tra questi due termini - "silenzio" e "rumore" - a riassumere le idee di "contesto sonoro piacevole" e viceversa "inquinato, fastidioso", portando con sé l'erronea convinzione secondo cui per realizzare un paesaggio sonoro di qualità sia sufficiente ridurre il volume dei suoni che lo caratterizzano ${ }^{6}$.

Le scelte che sostengono la "costruzione del silenzio" nel Morelli mostrano come il significato positivo di questo elemento sia reso possibile da una specifica interpretazione del contesto ambientale, dove l'evoluzione del luogo emerge dalla selezione di alcuni suoni piuttosto che di altri. Una lezione ancora attuale, che si lega a una corretta lettura del rapporto uomo-ambiente, in un orizzonte percettivo, sociale e culturale locale (Sim 2008).

$\grave{E}$ probabilmente questa caratteristica a segnare in modo evidente la continuità tra l'idea di "cura" medica, dove il suono appare come uno dei fattori di guarigione, e un processo di "cura" - heideggerianamente parlando - che deve instaurarsi tra individuo e territorio. Da un lato si delinea un percorso che implica la messa in discussione dell'equilibrio psico-fisico del malato, non soltanto in senso farmacologico. Dall'altro la capacità del paziente di stabilire relazioni virtuose con gli elementi dell'ambiente: a partire dal distacco dai ritmi frenetici e insalubri della civiltà industriale, approdando alla capacità di entrare in sintonia con un luogo differente, senza reintrodurre in esso meccanismi "malati". Tra il soggetto e l'ambiente si instaura quindi una coesistenza virtuosa, che rappresenta la base per la "cura" della malattia in senso stretto.

${ }^{6}$ Anche alla luce dell'esperienza individuale, è piuttosto semplice dimostrare la fallacia di tale affermazione. Quante volte siamo portati ad "alzare il volume della radio" anche oltre il consentito, quando sentiamo un brano che ci piace, e quante volte condanniamo un suono sgradevole, per quanto di bassa intensità. Proprio una "passeggiata sonora" (Westerkamp 1974) condotta all'interno del parco del Morelli nel $2012 \mathrm{mi}$ ha fornito un esempio per argomentare tale affermazione. Addentrandomi in un tratto di bosco caratterizzato dalla presenza di una cascatella, ho avuto la sensazione di trovarmi in un luogo in qualche modo magico, incantato, estremamente tranquillo e rilassante. Ascoltando con più attenzione, mi sono reso conto che in realtà il suono dello scroscio dell'acqua rappresentava uno degli elementi di maggiore intensità di tutta la visita. In prossimità di alcuni padiglioni, al contrario, suoni di ventole, impianti di condizionamento e motori rendevano il paesaggio sonoro molto meno attraente, nonostante la minore intensità del volume acustico complessivo. Per quanto l'affermazione derivi da un approccio puramente fenomenologico e soggettivo, e non possa quindi essere supportata da evidenze scientifica (se non quella molto rudimentale di una misurazione manuale della pressione sonora attraverso una app per smartphone), la stessa sensazione mi è stata confermata dai partecipanti alla passeggiata. 


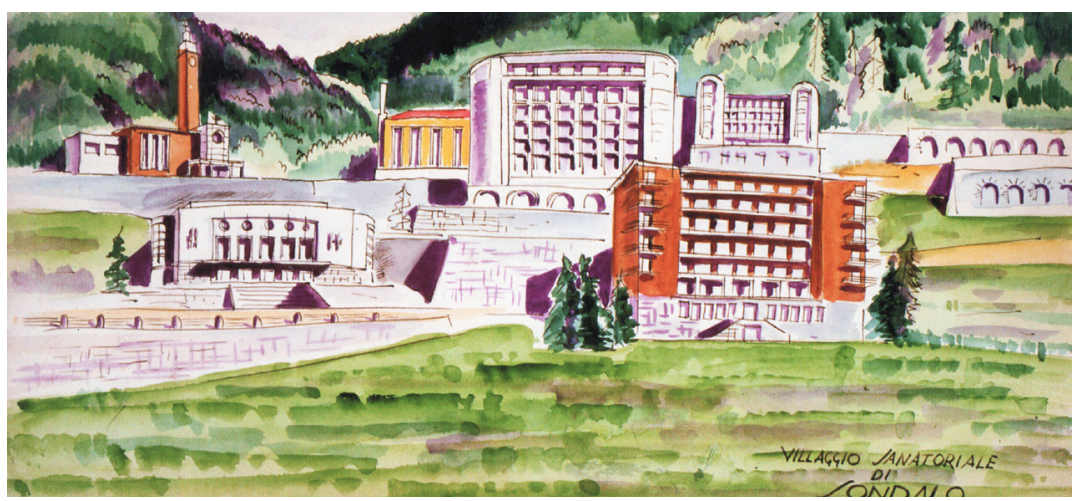

Figura 3. - Illustrazione di R. Mattiangeli del Panorama di progetto. Fonte: Collezione privata. L'immagine mostra il bilanciamento dei volumi, finalizzato a generare un senso di appartenenza e di micro-comunità all'interno del sanatorio. Sulla sinistra, in primo piano, si nota il teatro che sarebbe dovuto sorgere nel centro della piazza principale.

A fronte di una condizione abitativa come quella contemporanea, che richiede sempre meno attenzione per l'elemento acustico, il percorso sanatoriale invita l'individuo verso un "silenzio" che non è esito involontario e residuale di interazioni casuali, ma il prodotto di una attenta selezione di elementi, portata avanti nel tempo, segno del lungo confronto tra l'attività umana e il territorio (Rocca 2019).

Se non compreso all'interno di questo scenario, il rischio diventa quello di associare la condizione acustica che abbiamo descritto a un'idea di "naturalità" del tutto idealizzata: che ben poco avrebbe a che fare con il senso di questo luogo, e tantomeno potrebbe rappresentare una risposta per la maggior parte dei nostri ambienti abitati. Un riferimento che spingerebbe verso una contrapposizione radicale e inconciliabile tra ciò che è prodotto dall'uomo e ciò che invece rimane "incontaminato". All'interno del paesaggio del Morelli, nulla è generato da una natura realmente libera di esprimere se stessa. Ogni area verde, così come ogni elemento del parco, è pensato dall'uomo all'interno di un disegno artificiale riconoscibile. Ciò che conferisce qualità all'ambiente sonoro non è quindi l'assenza dell'uomo, ma l'equilibrata relazione tra artificio e contesto, che genera complessità, variabilità acustica e conferisce qualità al paesaggio (Turri 2004).

Il "silenzioso" paesaggio si fa dunque segno critico rispetto a una condizione percettiva in cui l'uomo perde contatto con la qualità del 
suono che lo circonda. Normalmente immersi in luoghi privi di valore e di significato, siamo sempre più spinti a trascurare la relazione in atto con i luoghi in cui viviamo, accontentandoci di realizzare attraverso dispositivi tecnologici gli sfondi ideali e personalizzati della nostra vita. Tale disattenzione favorisce l'uniformazione e l'omologazione dei contesti collettivi, generando dei veri e propri circoli viziosi.

Di fronte a queste derive, il Morelli ci ricorda che il progetto e la conservazione del paesaggio sonoro devono operare a vantaggio della costruzione di una relazione tra la sensibilità degli utenti e i linguaggi, i rit$\mathrm{mi}$, le pause e le alternanze locali. Solo questa intenzione potrà produrre una reale identificazione tra le persone e i luoghi, innescando delle azioni di cura e di valorizzazione del territorio.

\section{RIFERIMENTI BIBLIOGRAFICI}

Bonesio, L. and Del Curto, D. eds. 2011. Il villaggio Morelli. Identità paesaggistica e patrimonio monumentale. Sondrio: Diabasis.

Bonesio, L., D. Del Curto, G. Menini eds. 2012. Una questione di paesaggio. Il villaggio sanatoriale Morelli e la Valtellina. Sesto San Giovanni: Mimesis.

Cage, J. 1961. Silence. Wesleyan: University Press.

Dorfles, G. 2008. Horror Pleni: l'inciviltà del rumore. Roma: Castelvecchi.

Filiberti, F. ed. 2007. Il silenzio. Novara: Interlinea.

Foucault, M. 1993. Sorvegliare e punire. Nascita della prigione, Einaudi: Torino.

Lombardi, D. 2003. Rumori futuri, studi e immagini sulla musica futurista, Firenze: Vallecchi.

Mocchi, M. 2020. Città di suono. Per un incontro tra architettura e paesaggio sonoro. Siracusa: LetteraVentidue.

Pivato, S. 2011. Il secolo del rumore. Bologna: Il Mulino.

Rocca, L. ed. 2019. I suoni dei luoghi. Percorsi di geografie degli ascolti. Milano: Carocci.

Schafer, M. 1985. Il paesaggio sonoro. Milano: Ricordi LIM.

Sim, S. 2008. Manifesto per il silenzio. Torino: Feltrinelli.

Turri, E. 2004. Il paesaggio e il silenzio. Venezia: Marsilio.

Volcler, J. 2012. Il suono come arma. Gli usi militari e polizieschi dell'ambiente sonoro. Roma: DeriveApprodi.

Westerkamp, H. 2007. "Soundwalking”, in Carlyle A. ed., Autumn Leaves. Sound and Environment in Artistic Practice. Paris: Double Entendre. 
